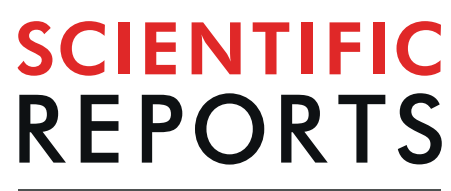

\title{
Study on collagen parameters in vulvar cancer and preneoplastic lesions by Second Harmonic Generation microscopy
}

\author{
Maria das Graças de Fátima Cavalcanti Castor ${ }^{1,6}$, Leuridan Cavalcante Torres ${ }^{1,2 *}$, \\ Roberto José Vieira de Mello ${ }^{3}$, Rodrigo de Andrade Natal ${ }^{4,6}$ \& José Vassallo ${ }^{4,5}$
}

The extracellular matrix plays an important role in cellular balance, and collagen fibers are its most important component. Over the last few years, second harmonic generation (SHG) microscopy has been used for the analysis of collagen fibers in several types of gynaecological cancers, such as breast and ovarian cancer. The value of collagen parameters obtained with this technique to gain insights on the physiopathology and on the prognostic evaluation of cancer has been advocated. Herein, we have characterized the collagen fibers in squamous cell carcinoma (VSCC) and preneoplastic lesions using the SHG microscopy. Collagen parameters, quantity, organization, and uniformity, of VSCC, adjacent skin of VSCC or preneoplastic lesions were compared with values obtained in normal tissue of healthy control. There was an evident decrease in the values of collagen fiber parameters in the VSCC. Increased quantity and uniformity of tumor associated collagen fibers were associated with the presence of lymph node metastases, which suggest a prognostic value of such parameters in the evaluation of vulvar cancer.

Vulvar cancer is the fourth leading gynaecological cancer worldwide ${ }^{1}$, following uterine corpus, ovarian, and cervical cancer. It represents only about $5 \%$ of all tumors of the lower genital tract ${ }^{1,2}$ and is thus considered a rare cancer. Among the different histological types of vulvar cancer, squamous cell carcinoma (VSCC) is the most common type (95\%), followed by melanoma ( $<5 \%)$, sarcoma (1-2\%), and basal-cell carcinoma (2\%). VSCC has two mechanisms of development, which arise via preneoplastic lesions called vulvar intraepithelial neoplasia (VIN): (1) infection with the human papillomavirus (HPV), which affects younger women ( $<50$ years), and (2) HPV-independent development (approximately 80\%), which appears after menopause (50-80 years) and originates from lichen sclerosus ${ }^{3-6}$.

VIN is graded in a similar way as cervical intraepithelial neoplasia: VIN 1, VIN 2, or VIN 3. In VIN 1, atypical cells are limited to the lower third of the vulvar squamous epithelium; in VIN 2, atypical cells compromise the deeper two thirds of the epithelium; and in VIN 3, atypical cells affect the entire squamous epithelium. In all three grades, the basal membrane remains intact, i.e., the lesions are not invasive. VIN 1 is not considered a precursor of vulvar cancer, as it has low malignant potential and low probability of evolving into VIN 2 and VIN 3 . These last two grades instead are considered high-grade lesions with real risk to transform into malignancy 7,8 .

Over the last few years, studies on cancer development have focused on the role of remodeling of the extracellular matrix (ECM), more specifically on collagen fibers (COL), to tumor progression. COL are major components of the ECM and are important for the maintenance of tissue and organ structure and integrity ${ }^{9,10}$. However, its role surpasses the structural aspect, and it is considered to influence tumor invasiveness and metastasizing ${ }^{9,10}$.

The study of COL in histology laboratories goes from the traditional stains, such as Masson's trichrome, Movat pentachrome, and picrosirius red staining, to the more specific detection methods, as immunohistochemistry,

\footnotetext{
${ }^{1}$ Division of Pelvis, Hospital de Cancer de Pernambuco, Recife, PE, Brazil. ${ }^{2}$ Translational Research Laboratory C. A. Hart, Instituto de Medicina Integral Prof. Fernando Figueira (IMIP), Recife, PE, Brazil. ${ }^{3}$ Division of Anatomic Pathology, Hospital de Cancer de Pernambuco, Recife, PE, Brazil. ${ }^{4}$ Laboratory of Investigative Pathology, CIPED, Faculdade de Ciências Médicas, UNICAMP, Campinas, SP, Brazil. ${ }^{5}$ Department of Anatomic Pathology, AC Camargo Cancer Center, São Paulo, Brazil. ${ }^{6}$ These authors contributed equally: Maria das Graças de Fátima Cavalcanti Castor and Rodrigo de Andrade Natal. *email: leuridan.torres@gmail.com
} 


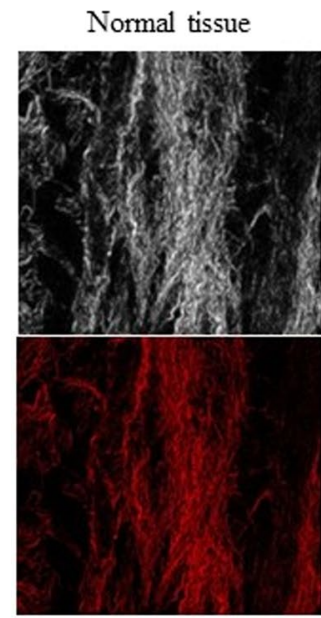

(RGB image)
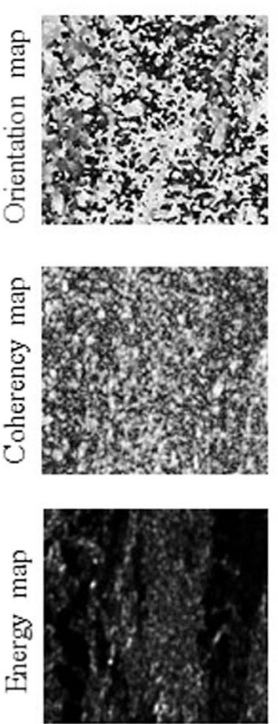

Normal tissue

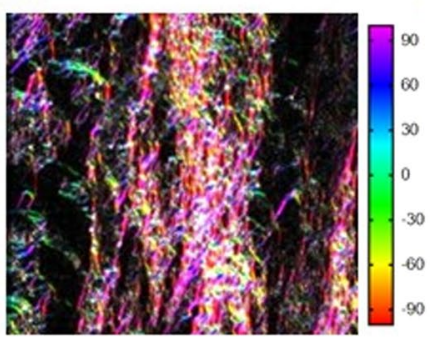

HSB color map

Hue: orientation

Saturation: Coherency

Brightness: Original image

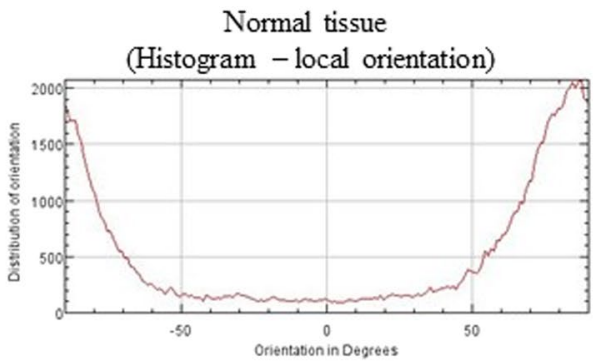

Figure 1. Results of the OrientationJ, the imageJ plug-in developed to get the histogram of local angles, the orientation, energy (quantity) and coherency (uniformity) maps of an image and the HSB color-coded map of normal tissue of healthy control.

the wide-field-of-view polarization, and the second harmonic generation (SHG) imaging. The SHG has been increasingly being used to study COL, due to its high sensitivity and specificity. It is a quantitative nonlinear optical microscopy technique that captures images from biological tissues having polarized anisotropic structures, such as $\mathrm{COL}^{11,12}$.

The association of increased COL expression in vulvar carcinoma and higher tumor aggressiveness has been recently reported, but the evaluation of COL was made using the picrosirius staining ${ }^{13}$. In the present study, SHG images were used to determine the quantity, uniformity, and organization of COL in vulvar cancer and its preneoplastic lesions. It is intended to determine the clinical value of COL evaluation by this method, not previously reported in vulvar neoplasia.

\section{Results}

Collagen fiber parameters in VSCC. We made the OrientationJ plug-in and an ImageJ for angle analysis the orientation, energy, and coherency maps, hue-saturation-brightness (HSB) color-coded map and orientation histogram in normal tissue of healthy control (individual without VSCC or VIN; Figure 1), VIN 3 (Figure 2) and adjacent skin of VSCC (Figure 3) and VSCC (Figure 4). Images show the number of collagen fibers arranged at different angles and tissues. It is observed that normal tissue of healthy control presents the better organization of collagen fibers, demonstrated by the peak between angles $60^{\circ}$ and $90^{\circ}$ (Figure 1). In the analysis of VIN 3 and adjacent skin of VSCC, we observed the highest amount of collagen fibers compared to VSCC (Figures 2 and 3, respectively). It is observed peaks in the number of collagen fibers at various angles in VSCC, showing that the region presents an absence of collagen fibers organization (Figure 4). Collagen quantity and uniformity parameters were significantly higher in group of VSCC patients with distant metastatic compared to without metastasis $(\mathrm{p}=0.01$ and $\mathrm{p}=0.02$, respectively; Figure 5$)$.

The analysis of the collagen fibers revealed decreased quantity, uniformity, and organization in intratumoral stroma compared to adjacent skin of VSCC ( $p<0.05$; Table 1$)$. According to the tumoral topography (labia minora, labia majora, and both), the quantity, uniformity, and organization of collagen parameters of labia minora were decrease compared to labia majora and in both labia $(\mathrm{p}=0.008, \mathrm{p}=0.01$, and $\mathrm{p}=0.005$, respectively) (Table 1$)$. The quantity, uniformity, and organization of collagen fibers were reduced in the intratumoral stroma when compared to adjacent skin in labia minora and labia majora in the VSCC $(\mathrm{p}<0.05$; Table 2$)$.

\section{Discussion}

COL parameters are reduced in VSCC and VIN in comparison to dermis adjacent to neoplasia. However, no significant difference was observed comparing COL parameters between VSCC and VIN patients. Additionally, patients with metastatic VSCC presented higher COL parameters than those without metastases. These findings agree with our previous study on luminal breast cancer, in which intratumoral COL parameters were reduced in comparison to peritumoral areas ${ }^{14}$. Similarly, patients with parameters associated with more aggressive disease, 


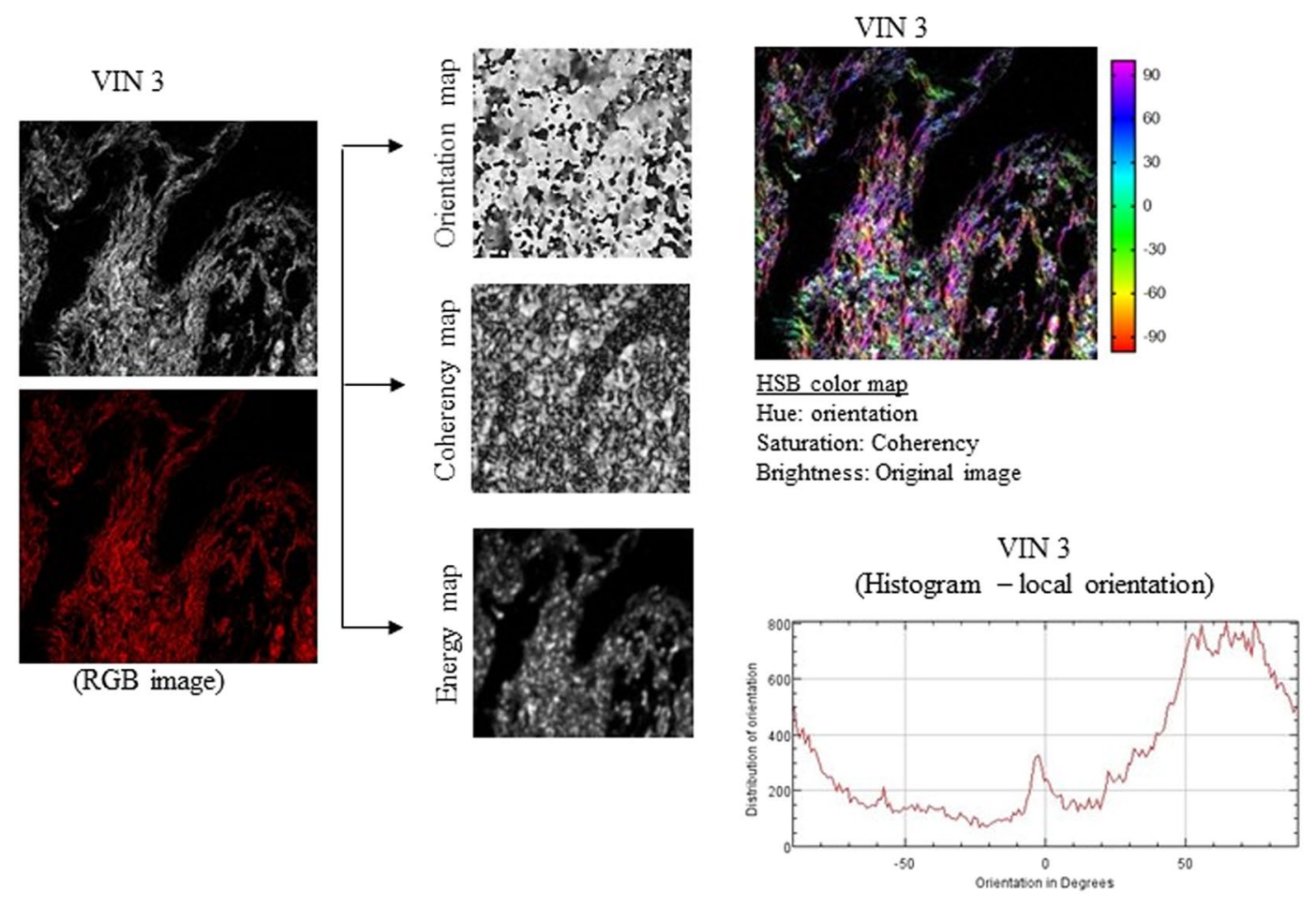

Figure 2. Results of the OrientationJ, the imageJ plug-in developed to get the histogram of local angles, the orientation, energy (quantity) and coherency (uniformity) maps of an image and the HSB color-coded map of VIN 3.

Adjacent skin of VSCC
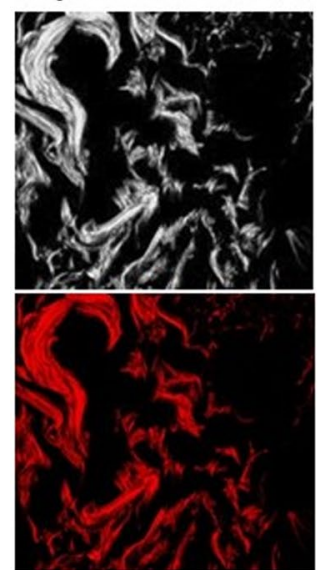

(RGB image)
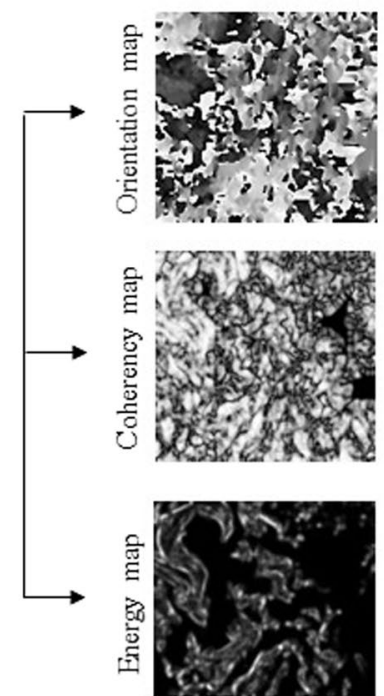

Adjacent skin of VSCC

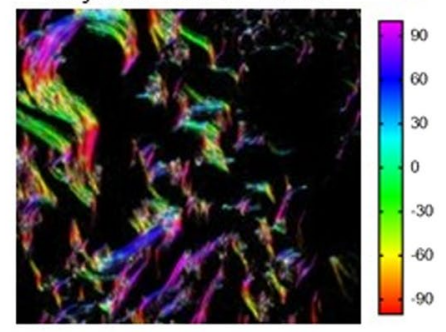

HSB color map

Hue: orientation

Saturation: Coherency

Brightness: Original image

Adjacent skin of VSCC (Histogram - local orientation)

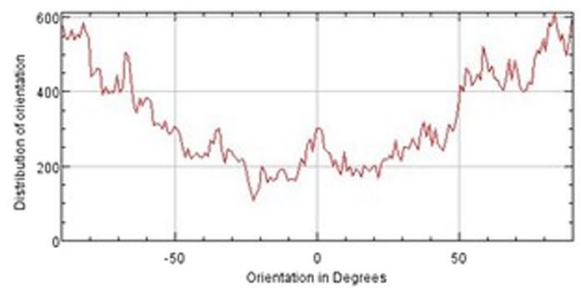

Figure 3. Results of the OrientationJ, the imageJ plug-in developed to get the histogram of local angles, the orientation, energy (quantity) and coherency (uniformity) maps of an image and the HSB color-coded map of adjacent skin of vulvar squamous cell carcinoma (VSCC).

presented higher intratumoral COL values ${ }^{14}$. Using another method to evaluate COL expression, it was recently found that higher values in VSCC were associated to superior aggressiveness ${ }^{12}$.

SHG microscopy has been used over the last few years to analyze COL in several types of cancer, due to its high sensitivity and specificity ${ }^{11,12,15}$. In our study, we used SHG to study COL in vulvar cancer, which, although 

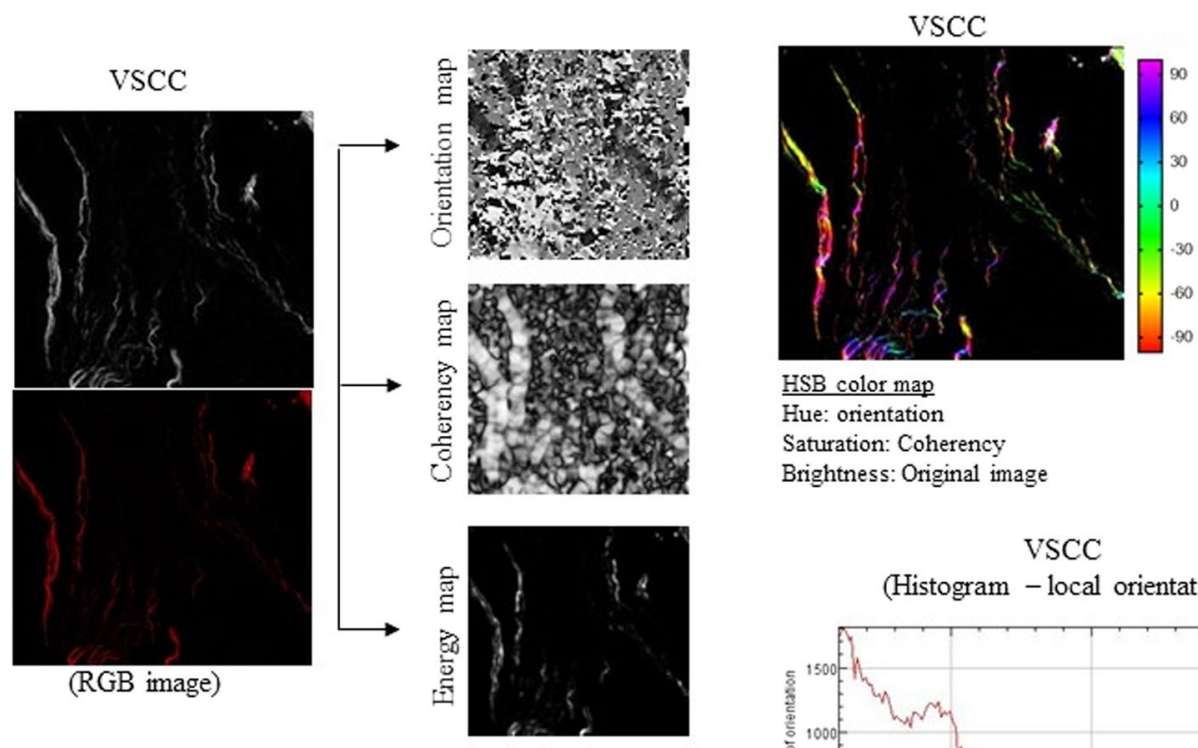

HSB color map

Hue: orientation

Saturation: Coherency

Brightness: Original image

\section{VSCC}

(Histogram - local orientation)

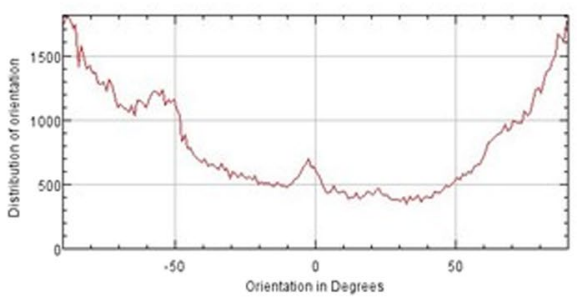

Figure 4. Results of the OrientationJ, the imageJ plug-in developed to get the histogram of local angles, the orientation, energy (quantity) and coherency (uniformity) maps of an image and the HSB color-coded map of vulvar squamous cell carcinoma (VSCC).

A)

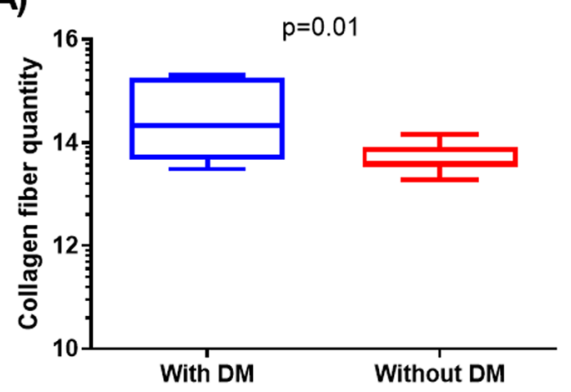

B)

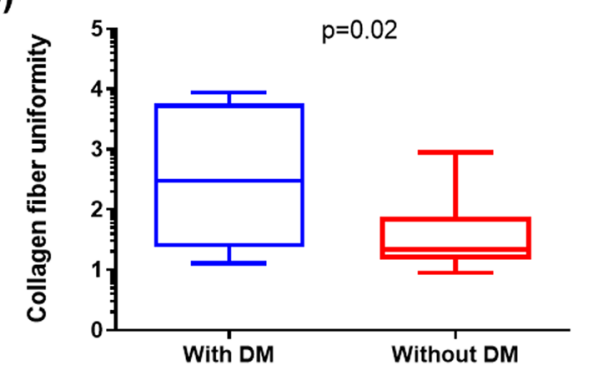

C)

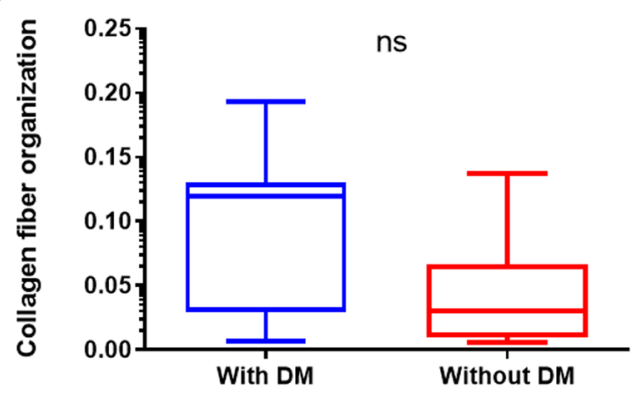

Figure 5. Comparative analysis of collagen parameters in vulvar squamous cell carcinoma (VSCC) in patients with distant metastasis (DM) and without DM: (A) Collagen Fiber quantity, (B) Collagen Fiber uniformity and $(\mathbf{C})$ Collagen Fiber organization. Ns: non-significant.

considered a rare cancer of elderly women, has been increasing in incidence in younger women over the last few decades $^{16-18}$. Studies on breast, head and neck, esophageal, and colorectal cancer using SHG reported that longer and denser COL in the tumor microenvironment was associated with tumor invasion ${ }^{9,10}$. Others have shown that the presence of tumor invasion was associated with higher COL quantity and uniformity, but lower organization ${ }^{19,20}$. 


\begin{tabular}{|c|c|c|c|c|c|c|c|}
\hline \multirow[b]{3}{*}{ Parameters } & \multicolumn{2}{|l|}{ VSCC } & \multirow[b]{3}{*}{ P-valor* } & \multicolumn{3}{|l|}{ Topography } & \multirow[b]{3}{*}{ P-valor** } \\
\hline & \multirow{2}{*}{\begin{tabular}{|l|} 
Intratumoral stroma \\
Median (IQR)
\end{tabular}} & \multirow{2}{*}{\begin{tabular}{|l|} 
adjacent skin \\
Median (IQR) \\
\end{tabular}} & & \multirow{2}{*}{\begin{tabular}{|l|} 
Labia minora \\
Median (IQR)
\end{tabular}} & \multirow{2}{*}{\begin{tabular}{|l|} 
Labia majora \\
Median (IQR) \\
\end{tabular}} & \multirow{2}{*}{\begin{tabular}{|l|} 
In both labia \\
Median (IQR)
\end{tabular}} & \\
\hline & & & & & & & \\
\hline Quantity & $13.90(13.55-15.10)$ & $16.10(15.73-16-48)$ & $<0.0001$ & $13.53(13.4-13.8)$ & $14.43(13.7-15.3)$ & $14.13(13.6-15.9)$ & 0.008 \\
\hline & & & & & & & 0.011 \\
\hline Organization & $0.064(0.014-0.17)$ & $0.21(0.11-0.24)$ & 0.03 & $0.02(0.003-0.06)$ & $0.10(0.02-0.19)$ & $0.05(0.02-0.10)$ & 0.005 \\
\hline
\end{tabular}

Table 1. Collagen fibers parameters of intratumoral area and adjacent skin of vulvar squamous cell carcinoma (VSCC), and according to the tumoral topography. IQR: interquartile; *Mann Whitney test; **Kruskal-Wallis test. $\mathrm{p}<0.05$.

\begin{tabular}{|c|c|c|c|c|c|c|}
\hline \multirow[b]{3}{*}{ Parameters } & \multicolumn{2}{|l|}{ VSCC - Labia minora } & \multirow[b]{3}{*}{ P-valor* } & \multicolumn{2}{|l|}{ VSCC - Labia majora } & \multirow[b]{3}{*}{ P-valor* } \\
\hline & Intratumoral stroma & adjacent skin & & Intratumoral stroma & adjacent skin & \\
\hline & Median (IQR) & Median (IQR) & & Median (IQR) & Median (IQR) & \\
\hline Quantity & $13.54(13.4-14.02)$ & $15.73(15.6-17.1)$ & 0.001 & $14.43(13.6-15.3)$ & $16.7(16.1-16.4)$ & $<0.0001$ \\
\hline Uniformity & $1.25(1.09-1.90)$ & $4.06(3.94-5.89)$ & 0.0003 & $2.55(1.42-3.68)$ & $4.68(4.55-5.22)$ & 0.0005 \\
\hline Organization & $0.033(0.006-0.054)$ & $0.17(0.14-0.29)$ & 0.001 & $0.10(0,02-0.19)$ & $0.21(0.20-0.24)$ & 0.004 \\
\hline
\end{tabular}

Table 2. Collagen fibers parameters of intratumoral stroma and adjacent skin of vulvar squamous cell carcinoma (VSCC) in the labia minora and labia majora. IQR: interquartile; *Mann Whitney test. $\mathrm{p}<0.05$.

When comparing lesions according to the anatomical site, the labia minora showed a decreased quantity, uniformity, and organization of intratumor COL in relation to the labia majora. This difference might be due to the heterogeneous composition of both tissues, one more akin to the mucosa and the other, to the skin.

The incidence of vulvar cancer is highest in elderly, post-menopausal women, in whom decreased estrogen production may be associated with a negative effect on cutaneous turgor, loss of skin elasticity ${ }^{21}$ and reduced immune defences ${ }^{22,23}$. It could be hypothesized that the recurrent inflammation due to these predisposing factors might play a role in the development of vulvar cancer. Although the tissues adjacent to our patient's tumors by histological examination were considered tumor-free margins, it was observed that on visualization of images obtained by SHG microscopy, these tissues showed small changes in COL parameters when compared to normal tissue in healthy women. Normal tissue was used for comparative analysis of the normal tissue image adjacent to the tumor by microscopy. We know that the free margin of the tumor is very important for locoregional control of the vulvar tumor, because when compromised, it is usually indicative for performing a re-excision ${ }^{24}$. This suggests that SHG microscopy may be an important meteorology for safely distinguishing areas that are tumor-free margins from those with very early precursor lesions.

The present study has some limitations. The number of cases is relatively small, due the uni-institutional design. However, it represents an infrequent cancer, and encouraging data were obtained on COL quantification in this setting. Another limitation is represented by the fact that the controls used were areas in the same samples, without microscopically detectable neoplasia. There is a potential that finer alterations at the molecular level could have interfered with the results, but a significant difference has been achieved between most parameters from tumor versus non-tumor areas. The use of SHG can be considered a drawback, because of the costs of the equipment and difficulty in managing it. However, it should be acknowledged that this cost has been decreasing over the last few years, what may allow SHG to become included in the diagnostic practice in the near future ${ }^{11,12}$.

Our findings on vulvar neoplasia add information on COL quantification by SHG microscopy in vulvar carcinoma and corroborate the general results with other tumors studied. They reinforce the notion that COL quantity and distribution play an important role in tumor progression. Further, it is shown herein that alterations in COL are precocious in vulvar carcinogenesis, as the same alterations were found already in VIN.

\section{Methods}

Experimental design and participants. An analytical and retrospective study was performed between 2000 and 2010, after approval by the Human Research Ethics Committee of HCP - Number of Certificate of Presentation for Ethical Appreciation is 50720115.9.0000.5205. The guidelines followed the resolution number $466 / 12$ of the National Health Council of Brazil for involving human participants. The informed consent was obtained from all subjects. The study was executed at the Cancer Hospital of Pernambuco (HCP, Pernambuco), and the Multiuser Laboratory of the "Gleb Wataghin" Physics Institute (INFABiC - State University of Campinas Unicamp, São Paulo). A survey was undertaken on patient records with the diagnoses of VSCC and VIN, between 2000 and 2010. The formalin fixed paraffin embedded tissue blocks from the patients with both diagnoses were retrieved from the archives of the Pathology Department of Hospital de Cancer de Pernambuco, Recife, Brazil.

The study included a total of 52 Brazilian patients older than 18 years, of which 40 patients had been diagnosed with VSCC and 12 with potential precursor lesions (VIN 2 and VIN 3). Adjacent tissues to the tumor with no neoplastic and/or preneoplastic morphological features were used as controls (17 patients). The metastases considered in the study were all to the lymph nodes, confirmed by histopathology of the surgical specimens (seven patients; Table 3). Clinicopathological data were obtained from the patients' files and from the pathological reports, and included age, topography, and presence of metastases (Table 3). All H\&E stained slides were reviewed 


\begin{tabular}{|c|c|c|}
\hline PARAMETERS & VSCC & VIN 2-3 \\
\hline & $\mathrm{N}=\mathbf{4 0}$ & $\mathbf{N}=12$ \\
\hline & $\mathbf{N}(\%)$ & $\mathbf{N}(\%)$ \\
\hline \multicolumn{3}{|l|}{ AGE (years) } \\
\hline$<60$ & $9(22.5)$ & $7(58.3)$ \\
\hline$\geq 60$ & $31(77.5)$ & $5(41.7)$ \\
\hline \multicolumn{3}{|c|}{ DISTANT METASTASIS (lymph node+) } \\
\hline Yes & $7(17.5)$ & 0 \\
\hline No & $33(82.5)$ & $12(100)$ \\
\hline \multicolumn{3}{|l|}{ LESION LOCATION } \\
\hline Labia Minora & $10(25.0)$ & $3(25.1)$ \\
\hline Labia Majora & $17(42.5)$ & $2(17.7)$ \\
\hline In both labia (Minora and Majora) & $12(30.0)$ & $5(41.7)$ \\
\hline Clitoris and pubis & $1(2.5)$ & $2(16.7)$ \\
\hline
\end{tabular}

Table 3. Clinical characteristics of patients with vulvar squamous cell carcinoma (VSCC) and vulvar intraepithelial neoplasia (VIN).

by the pathologists and the areas of interest were selected for studies using the second harmonic generation microscopy (SHG).

Second harmonic generation (SHG). Slides stained with H\&E were submitted to the identification and selection of collagen (COL) in the stromal areas adjacent to VIN, in intratumoral stroma, and in normal adjacent tissues. In each case, three areas were selected, and these areas were submitted to SHG analyses at the INFABiC Unicamp ${ }^{14}$.

SHG is a nonlinear optical process, in which two photons with the same frequency interact with a nonlinear material and generate a new photon with twice the energy and half the wavelength of the initial photons. Fibrillar collagen has high nonlinear susceptibility, with SHG being the ideal method to evaluate its structure ${ }^{12,13}$. SHG microscopy was performed on a confocal microscope Zeiss LSM 780-NLO (Carl Zeiss AG, Göttingen, Germany) using a lens with an objective of $40 \times / 1.3$ EC Plan-Neofluar with immersion oil. The highest numerical aperture (1.3) was necessary to allow an adequate spatial resolution to observe the fibrils. The excitation wavelength was $780 \mathrm{~nm}$, with a pulse of approximately $100 \mathrm{fs}$ at a repetition frequency of $80 \mathrm{MHz}$, supplied by a Tsunami Ti:Sapphire laser (Spectra-Physics, Irvine, CA, USA). The objective lens laser power was approximately $80 \mathrm{~mW}$, with circular polarization. The acquisition time for each image was approximately $60 \mathrm{~s}$.

Only the SHG forward signal at $390 \mathrm{~nm}$ was collected using a condenser lens $0.55 \mathrm{NA}-\mathrm{WD} 26 \mathrm{~mm}$. A short-pass SP690 (Omega Filters, Brattleboro, VT, USA) was used to filter the excitation wavelength at $780 \mathrm{~nm}^{25}$; this was followed by filtering with long-pass filters LP490 at $45^{\circ}$ to avoid two-photon excited fluorescence (TPEF), and SP430 at $90^{\circ}$ to filter only the SHG signal. Two detectors were used to capture the TPEF and SHG signals, namely a photomultiplier tube detector (PMT) and non-descanned detector (NDD), respectively. Of these, only the SHG signal was considered for the present study. The emission spectrum analysis between 350 and $430 \mathrm{~nm}$ showed only a narrow peak at $390 \mathrm{~nm}$ consistent with the SHG signal. The TPEF signal given by eosin could also be detected at $530 \mathrm{~nm}$.

The procedure described by Burke et al. ${ }^{25}$ was used, with daily optical alignments performed by obtaining a normalization factor, which consisted of the SHG image of a standard specimen (i.e., a histological section of human aorta), at the beginning of each experiment. The vision field of this objective was $212 \times 212 \mathrm{~cm}$, but to avoid the figure margins in which the SHG intensity was weaker, we used a zoom to select a more homogeneous area of $177 \times 177 \mathrm{~cm}$. To eliminate background signals, a "blind" observer, with a degree in physics and without medical training, applied a common threshold to all images, comparing them with the control sample of human aorta. After this, all background signals unrelated to COL were eliminated.

SHG signals were stratified according to COL organization and structure in the stroma, using image-pattern analysis methods. Quantitative analysis of COL parameters was performed on SHG images using ImageJ (http:// imagej.nih.gov/ij/) and the OrientationJ plug-in software ${ }^{26}$. Nine representative areas $(150 \times 150$ pixels $)$ were randomly selected from each image to evaluate COL quantity, uniformity, and organization, as described below.

COL quantity was determined as the sum of SHG signal intensities in $1024 \times 1024$ pixel images, which was directly related to the quantity of collagen molecules. These values were obtained using the integrated density function of ImageJ software. Using the OrientationJ plug-in, as previously described ${ }^{26}$, we calculated COL uniformity (energy) and organization (coherence). Uniformity (energy) reflects the density of COL, i.e., whether fibers are farther from (lower values) or closer to (higher values) each other. Organization (coherence) reflects the spatial direction of COL, with values between 0 (completely isotropic areas, with zero directionality) to 1 (highly oriented fibers). From the perspective of mathematical definitions, these parameters should be correlated. The relationship between COL parameters for vulvar lesions, normal adjacent tissues, and VIN was added to the calculation $^{27}$.

Statistical analyses. Descriptive statistics for categorical variables are presented in tables as the distribution of absolute and relative frequencies, whereas continuous variables are presented as measures of central tendency (i.e., median). Normality was tested using the D'Agostino-Pearson test. The variables quantity and uniformity of collagen fibers were log-transformed, because they depend upon the square of the SHG signals. Continues values 
were plotted as the median and interquartile range (25th-75th). The Mann-Whitney nonparametric test was used to analyze numerical variables between two groups. The Kruskal-Wallis test was used to analyze numerical variables between three groups. Data analysis was performed using GraphPad Prism v 8.0 software (GraphPad Software, San Diego, CA). All comparisons were considered statistically significant at $\mathrm{p}<0.05$.

\section{Data availability}

Data sharing, evidence of data sharing and peer review of data required.

Received: 16 September 2019; Accepted: 24 February 2020;

Published online: 27 March 2020

\section{References}

1. Wang, Z. et al. The clinicopathological and prognostic impact of 14-3-3 sigma expression on vulvar squamous cell carcinomas. BMC Cancer. 8(1). 2008.

2. Van de Nieuwenhof, H., van der Avoort, I. \& de Hullu, J. Review of squamous premalignant vulvar lesions. Critical Reviews in Oncology/Hematology. 68(2), 131-156 (2008).

3. Van de Nieuwenhof, H. et al. Differentiated vulvar intraepithelial neoplasia is often found in lesions, previously diagnosed as lichen sclerosus, which have progressed to vulvar squamous cell carcinoma. Modern Pathology. 24(2), 297-305 (2011).

4. Ueda, Y. et al. Two Distinct Pathways to Development of Squamous Cell Carcinoma of the Vulva. Journal of Skin Cancer 2011, 1-7 (2011).

5. Alkatout, I. et al. Vulvar cancer: epidemiology, clinical presentation, and management options. International Journal of Women's Health. 7, 305-313 (2015).

6. Chokoeva, A. et al. Vulvar cancer: a review for dermatologists. Wiener Medizinische Wochenschrift. 165(7-8), 164-177 (2015).

7. Firenczy, A. Vulvar intraepithelial neoplasia. In: Apgar, B. S, Brotzman, G. L \& Spitzer, M. Colposcopy: principle sand practice. Philadelphia: WB Sunders Company. (1) 1-22 (2002).

8. Sideri, M. et al. Squamous Vulvar Intraepithelial Neoplasia: 2004 Modified Terminology, ISSVD Vulvar Oncology Subcommittee. Obstetrical \& Gynecological Survey. 61(3), 174-175 (2006).

9. Hanley, C. et al. A subset of myofibroblastic cancer-associated fibroblasts regulate collagen fiber elongation, which is prognostic in multiple cancers. Oncotarget. 7(5), 6159-6174 (2015).

10. Brooks, M. et al. Positive association of collagen type I with non-muscle invasive bladder cancer progression. Oncotarget.; 7(50) (2016).

11. Drifka, C. R. et al. Comparison of Picrosirius Red Staining With Second Harmonic Generation Imaging for the Quantification of Clinically Relevant Collagen Fiber Features in Histopathology Samples. 64(9) 519-529 (2016).

12. Mostaço-Guidolin, L., Rosin, N. \& Hackett, T. Imaging Collagen in Scar Tissue: Developments in Second Harmonic Generation Microscopy for Biomedical Applications. International Journal of Molecular Sciences. 18(12), 1772 (2017).

13. Holthoff, E. R. et al. Vulvar squamous cell carcinoma aggressiveness is associated with differential expression of collagen and STAT1. Clin Proteomics.; 14: 40 Dec 6 (2017).

14. Natal, R. A. et al. Collagen analysis by second-harmonic generation microscopy predicts outcome of luminal breast cancer. Tumour Biol.; 40(4) Apr (2018).

15. Calleja-Agius, J., Brincat, M. \& Borg, M. Skin connective tissue and ageing. Best Practice \& Research Clinical Obstetrics \& Gynaecology. 27(5), 727-740 (2013).

16. Adur, J. et al. Recognition of serous ovarian tumors in human samples by multimodal nonlinear optical microscopy. Journal of Biomedical Optics. 16(9), 096017 (2011).

17. Joura, E., Lösch, A., Haider-Angeler, M., Breitenecker, G. \& Leodolter, S. Trends in vulvar neoplasia. Increasing incidence of vulvar intraepithelial neoplasia and squamous cell carcinoma of the vulva in young women. The Journal of reproductive medicine. 45(8), 613-615 (2000).

18. Saraiya, M. et al. Incidence of in situ and invasive vulvar cancer in the US, 1998-2003. Cancer supplement. 113(10), 2865-72 (2008).

19. Weiskopf, D., Weinberger, B. \& Gubeck-Loebenstein, B. The aging of imune system. Transpl. Int. 22, 1041-50 (2009).

20. Pointer, B. P. et al. Association of collagen architecture with glioblastoma patient survival. J Neurosurg. 126(6), 1812-1821 (2017).

21. Lanneau, G. et al. Vulvar cancer in young women: demographic features and outcome evaluation. American Journal of Obstetrics and Gynecology. 200(6), 645.e1-645.e5 (2009).

22. Raine-Fenning, N. J., Brincat, M. P. \& Muscat-Baron, Y. Skin aging and menopause: implications for treatment. Am. J. Clin. Dermatol 4, 371-378 (2003).

23. Siegel, R., Naishadham, D. \& Jemal, A. Cancer statistics, 2013. CA: A Cancer Journal for Clinicians. 63(1), 11-30 (2013).

24. Gasimli, K. et al. Impact of re-excision of residual adjacent vulvar intraepithelial neoplasia (VIN III) and histological tumour-free margin (hTFM) on survival in primary squamous cell carcinoma of vulva. Arch Gynecol Obstet.; 298(5):945-950 (Nov 2018).

25. Burke, K., Tang, P., Brown, E. Second harmonic generation reveals matrix alterations during breast tumor progression. J. Biomed. Opt. 18:31106. Mechanobiol.; 11:461-73 2013 (2012).

26. Rezakhaniha, R. et al. Experimental investigation of collagen waviness and orientation in the arterial adventitia using confocal laser scanning microscopy. Biomech. Model.

27. Chen, W.-L. et al. Single-wavelength reflected confocal and multiphoton microscopy for tissue imaging. J. Biomed. Opt. 14, 54026 (2009).

\section{Acknowledgements}

The authors disclosed receipt of the following financial support for the research, authorship, and/or publication of this article: The authors thank the National Institute of Science and Technology on Photonics Applied to Cell Biology (INFABiC) at the State University of Campinas - Unicamp, São Paulo, Brazil, for access to equipment and assistance; INFABiC is co-funded by Fundação de Amparo à Pesquisa do Estado de São Paulo (FAPESP; grant no 08/57906-3) and Conselho Nacional de Desenvolvimento Científico e Tecnológico (CNPq; grant no $573913 / 2008-0)$.

\section{Author contributions}

Maria das Graças de Fátima Cavalcanti Castor and Rodrigo de Andrade Natal contributed equally in all steps of this article. M.G.F.C. and L.C.T. participated in the planning of the study, searched the data and the archival blocks of all patients, analyzed the results and wrote the paper. R.J.V.M., R.A.N. and J.V. reviewed the pathological slides, and selected the areas for S.H.G. analysis; R.A.N. performed the evaluation at the S.H.G. microscope; All authors critically read the paper and accepted the final version. 


\section{Competing interests}

The authors declare no competing interests.

\section{Additional information}

Correspondence and requests for materials should be addressed to L.C.T.

Reprints and permissions information is available at www.nature.com/reprints.

Publisher's note Springer Nature remains neutral with regard to jurisdictional claims in published maps and institutional affiliations.

(c) (i) Open Access This article is licensed under a Creative Commons Attribution 4.0 International (c) License, which permits use, sharing, adaptation, distribution and reproduction in any medium or format, as long as you give appropriate credit to the original author(s) and the source, provide a link to the Creative Commons license, and indicate if changes were made. The images or other third party material in this article are included in the article's Creative Commons license, unless indicated otherwise in a credit line to the material. If material is not included in the article's Creative Commons license and your intended use is not permitted by statutory regulation or exceeds the permitted use, you will need to obtain permission directly from the copyright holder. To view a copy of this license, visit http://creativecommons.org/licenses/by/4.0/.

(C) The Author(s) 2020 